\title{
Recent Sensitivity Pattern of Salmonella Typhi in a Private Hospital
}

\author{
KHAIRUL ISLAM, ${ }^{1}$ REAZ MAHMUD, ${ }^{2}$ MOSTOFA KAMAL CHOWDHURY, ${ }^{3}$ FAHIMA SHARMIN HOSSAIN, ${ }^{4}$ PRODIP \\ KUMAR BISWAS, ${ }^{5}$ SHYAMAL SARKER ${ }^{6}$
}

\begin{abstract}
:
Introduction: Enteric fever is highly prevalent in Bangladesh. Multidrug resistant salmonella Typhi is emerging throughout the world as well as in Bangladesh. Observing recent sensitivity pattern will help in choosing proper empirical antibiotics in resource limited country like Bangladesh as culture facilities is not available throughout the country.

Materials and Methods: The study included 86 S. typhi isolates from blood cultures of patients suffering from suspected typhoid fever who attended the outpatient clinics or were admitted in Islami Bank hospital in Dhaka during January 2015 to July 2017.

Results: A total of 86 S. typhi isolates were included in the study. Mean age of the study population was $22.06 \pm 17.1$ and there was male 44 (51.2\%) predominance. All the isolates of S. typhi were sensitive to Meropenem. Highest resistance was observed against Amikacin (62.4\%) closely followed by Co-timoxazole (48.4\%) and Amoxiclav (46.5\%). The S. typhi isolates showed low-level resistance against Ceftriaxone (1.16\%), Cefuroxime (4.6\%), Azithromycin(13.9\%), Levofloxacin (11.6\%).

Conclusions: According to findings of this study it can be advised that oral form of Cefuroxime, Levofloxacin and Azithromycin can be used as a first line. Then if patient is nonresponsive Ceftriaxone might be used. Meropeman should be reserved for selective cases.
\end{abstract}

Keywords: Typhoid, Multidrug resistance, Salmonella Typhi.

\section{Introduction:}

According to WHO the annual global incidence of typhoid fever is 21 million cases, of whom $1-4 \%$ end fatally. ${ }^{1}$ An estimated $90 \%$ of these deaths occur in Asia. On the Indian subcontinent, Pakistan has the highest incidence (451.7 per 100,000 persons/year) of typhoid fever followed by

1. Asst. Registrar (Medicine), Sarkari Karmachari Hospital, Dhaka.

2. Junior consultant (Medicine), Sarkari Karmachari Hospital, Dhaka.

3. Asst. Registrar, Dept. of Endocrinology, Dhaka Medical College Hospital, Dhaka.

4. Medical officer, Sarkari Karmachari Hospital, Dhaka.

5. Associate Professor of Medicine, Dhaka Medical College, Dhaka.

6. Professor of Medicine, Dhaka Medical College, Dhaka.

Corresponding author: Dr. Mostofa Kamal Chowdhury, Asst. Registrar, Dept. of Endocrinology, Dhaka Medical College Hospital, Dhaka. E-mail: mkcadil@yahoo.com. Mobile: 01711125938 .
India (214.2 per 100,000 persons/year). ${ }^{2}$ A community based study in an urban slum in Bangladesh, by Brook et al. suggested that the overall incidence was $3.9 / 1000$ persons/ear and the rate was higher in preschool children aged between 0 and 4 years (18.7 per 1000 persons/years). ${ }^{3}$ Increasing multidrug resistance of $\mathrm{S}$. typhi reduces the effective treatment options, increases treatment costs and results in higher rates of serious complications and death. ${ }^{1}$ In 1992, emergence of multidrug resistance enteric fever (resistance to Chloramphenicol, Ampicillin and Trimethoprim Sulfamethoxazole) was strongly addressed in Bangladesh; around $36.58 \%$ cases were reported in a large Study. ${ }^{4}$

Indiscriminate and injudicious use of antibiotics raises the risk of newer strains of resistant organisms. Uses of antibiotic should be guided by culture and sensitivity report. But in Bangladesh these facilities are not available throughout the country and poor people cannot afford. So in most of the cases physicians have to prescribe empirically. The recent sensitivity pattern will help them in choosing the most effective drug. It is also important to constantly monitor 
the susceptibility patterns of S. typhi, so as to provide suitable guidelines for treatment.

\section{Methods:}

The study included $86 \mathrm{~S}$. typhi isolates from blood cultures of patients suffering from suspected typhoid fever who attended the outpatient clinics or were admitted in Islami Bank hospital in Dhaka during January 2015 to July 2017.

\section{Bacterial Culture:}

Bile salt broth (broth culture) $)^{5}$ and streptokinase broth (clot culture $)^{6}$ blood samples were used for enrichment. The enriched samples after visible turbidity were streaked on Mac-Conkey, XLD and Wilson Blair media. The isolates producing characteristic colonies were identified by conventional biochemical tests using API20E and confirmed by agglutination with Salmonella O9, Vi specific and $\mathrm{Hd}$ antisera.

\section{Antimicrobial Susceptibility Testing:}

The antibiotic susceptibility testing was done by Kirby-Bauer disk diffusion method according to National Committee for Clinical Laboratory Standards (NCCLS) guidelines ${ }^{7}$ using Ampicillin (10 $\left.\mu \mathrm{g} / \mathrm{disk}\right)$, Amoxiclav, Azithromycin, Levofloxacin, Chloramphenicol (30 $\mu \mathrm{g} /$ disk), Co-trimoxazole (1.25-23.75 $\mu \mathrm{g} / \mathrm{disk})$, Ciprofloxacin $(5 \mu$ $\mathrm{g} /$ disk), Cefuroxime, Cefixime, Ceftriaxone ( $5 \mu \mathrm{g} /$ disk), Nalidixic acid (30 $\mu \mathrm{g} / \mathrm{disk})$ and Meropenem $(10 \mu \mathrm{g} / \mathrm{disk}){ }^{8}$ Escherichia coli ATCC 25922 was used as a negative control and S. typhi MTCC 734 was used as a positive control for the effectiveness of the antiotic disks. Commercially available six mm disks (Himedia Laboratories, Mumbai) were used. Isolates resistant to Ampicillin, Chloramphenicol and Co-trimoxazole were termed multidrug resistant (MDR).

\section{Results:}

A total of $86 \mathrm{~S}$. typhi isolates were included in the study. Mean age of the study population was $22.06 \pm 17.1$ and there was male $44(51.2 \%)$ predominance. Antibiogram of these isolates revealed that all the isolates of S. typhi were sensitive to Meropenem. Highest resistance was observed against Amikacin (62.4\%) closely followed by Co-timoxazole (48.4\%) and Amoxyclav (46.5\%). The S. typhi isolates showed low-level resistance against Ceftriaxone (1.16\%), Cefuroxime (4.6\%), Azithromycin (13.9\%), Levofloxacin (11.6\%). Approximately $24.4 \%(\mathrm{~N}=21)$ of the isolates were MDR.

\section{Table-I}

Number of S. typhi isolates resistant to antibiotics by disc diffusion method

\begin{tabular}{lcc}
\hline Antibiotic screened & No of resistant isolate & Percentage \\
\hline Amoxiclav & 40 & 46.5 \\
Azithromycin & 12 & 13.9 \\
Amikacin & 54 & 62.8 \\
Cefuroxime & 4 & 4.6 \\
Cefixime & 20 & 23.2 \\
Ceftriaxone & 1 & 1.16 \\
Ciprofloxacin & 30 & 34.8 \\
Co-trimoxazole & 42 & 48.4 \\
Gentamicin & 12 & 13.9 \\
Chloramphenicol & 28 & 32.5 \\
Levofloxacin & 10 & 11.6 \\
Meropenem & 0 & 0 \\
MDR & 21 & 24.4 \\
\hline
\end{tabular}

$\mathrm{MDR}=$ Multidrug resistant

\section{Discussion:}

Due to a combination of factors including poor sanitation and health care infrastructure, typhoid fever remains a major public health problem in most resource-poor countries such as Bangladesh. ${ }^{9}$ In most of the studies it had been showed that enteric fever mostly occurs in paediatric age group ${ }^{9,10}$ but in this study mean age of the patient was 22 years because of increased number of adult patients.

According to public and private hospital records, enteric fever is a major infectious disease occurring at high fluctuating incidences in this region..$^{9,10}$ The data presented in our study highlights that MDR exists in high percentage. The presence of MDR (i.e resistance to Ampicillin, Chloramphenicol and Co-trimoxazole) was $24.4 \%$. This finding is in accordance with other reports from same regions. ${ }^{4,9}$ Meropenem has no resistance. Ceftriaxone is still highly sensitive though it is costly and only found in injectable form. Among the oral form Cefuroxime, Levofloxacin and Azithromycin is most effective.

\section{Conclusions:}

Drug resistance develope due to injudicious and indiscriminate use of antibiotic. We should be careful in choosing antibiotics 
JM Vol. 19, No. 1

during empirical use. According to findings of this study it can be advised that oral form of Cefuroxime, Levofloxacin and Azithromycin can be used as a first line. Then if patient is nonresponsive Ceftriaxone might be used. Meropenem should be reserved for selective cases.

\section{Conflict of interest: None.}

\section{References:}

1. WHO, Weekly epidemiological record, No. 6. 2008;83:49-60.

2. Chiai RL, Acosta CJ, Danovaro-Holliday MC, Baiqing D, Bhattacharya SK, et al., A study of typhoid fever in five Asian countries: disease burden and implications for controls. Bull World Health Organ 2008;86(4):260-268.

3. Brook WA, Hossain A, Goswami D, Sharmeen AT, Nahar K, et al. Bacteremic typhoid fever in children in an urban slum, Bangladesh. Emerg Infect Dis 2005; 11(2):326-329.

4. Alam MN, Haq SA, Das KK, Mazid MN, Hassan Z, Ahsan SA, Ahmed N, Rahman KM, Siddiqui FM. Multidrug Resistant Enteric Fever in Bangladesh. Bangladesh J Med.1992;3(2):38-41.
Recent Sensitivity Pattern of Salmonella Typhi in a Private Hospital

5. Watson KC. Clot culture in typhoid fever. J Clin Patho 11954;7:305-307.

6. Watson KC. Laboratory and clinical investigation of recovery of Salmonella typhi from blood J Clin Microbio 11978;7:122-126.

7. Performance Standards for Antimicrobial disk Susceptibility Tests. CLSI, Wayne, PA, USA, 2005. NCCLS document M7-A.

8. Nath G, Tikoo A, Manocha H, Tripathi AK, Gulati AK. Drug resistance in Salmonella typhi in North India with special reference to ciprofloxacin. $\mathrm{J}$ Antimicrobial Chemother 2003;46:145-53.

9. Dewan AM, Corner R, Hashizume M, Ongee ET. Typhoid Fever and its Association with Environmental Factors in the Dhaka Metropolitan Area of Bangladesh: A Spatial and Time-Series Approach. PLoSNegl Trop Dis 2013;7(1):e1998.

10. Madhulika U, Harish BN, Parija SC. Current pattern in antimicrobial susceptibility of Salmonella Typhi isolates in Pondicherry. Indian $\mathrm{J}$ Med Res 2004;120:111-114. 